Strangeness production from SPS to LHC

This article has been downloaded from IOPscience. Please scroll down to see the full text article.

2008 J. Phys. G: Nucl. Part. Phys. 35104013

(http://iopscience.iop.org/0954-3899/35/10/104013)

View the table of contents for this issue, or go to the journal homepage for more

Download details:

IP Address: 192.84.145.254

The article was downloaded on 02/11/2012 at 09:57

Please note that terms and conditions apply. 


\title{
Strangeness production from SPS to LHC
}

\author{
F Becattini ${ }^{1}$ and J Manninen ${ }^{2}$ \\ ${ }^{1}$ Università di Firenze and INFN Sezione di Firenze, Via G Sansone 1, I-50019 Sesto F no, \\ Firenze, Italy \\ ${ }^{2}$ INFN Sezione di Firenze, Via G Sansone 1, I-50019 Sesto F no, Firenze, Italy \\ E-mail: becattini@fi.infn.it
}

Received 30 April 2008

Published 17 September 2008

Online at stacks.iop.org/JPhysG/35/104013

\begin{abstract}
Global strangeness production in relativistic heavy ion collisions at SPS and RHIC is reviewed. Special emphasis is put on the comparison with the statistical model and the canonical suppression mechanism. It is shown that recent RHIC data on strange particle production as a function of centrality can be explained by a superposition of a fully equilibrated hadron gas and particle emission from single independent nucleon-nucleon collisions in the outer corona.
\end{abstract}

\section{Introduction}

The enhancement of relative (to u,d quarks) strange quark production in high energy heavy ion collisions with respect to elementary collisions has been predicted a long time ago to be a signature of the quark gluon plasma (QGP) formation [1]. The idea was that chiral symmetry restoration favours strange quark production because of the reduced mass compared to its zero temperature constituent value. This abundant strangeness production could be observed provided that it survives hadronization, i.e. if the early produced strange quarks coalesce into hadrons without reannihilating. A specific prediction of such a mechanism is the enhancement of multiply strange particles, especially hyperons.

These phenomena have indeed been observed: the ratio of newly produced strange to u,d quarks (the so-called Wroblewski ratio $\lambda_{S}=\langle\mathrm{s} \overline{\mathrm{s}}\rangle / 2(\langle\mathrm{u} \overline{\mathrm{u}}\rangle+\langle\mathrm{d} \overline{\mathrm{d}}\rangle)$ shows about a factor 2 increase going from elementary to heavy ion collisions (see figure 1) as first observed in [2], and the hyperons show a clear hierarchical enhancement in central $\mathrm{Pb}-\mathrm{Pb}$ collisions with respect to peripheral $\mathrm{Pb}-\mathrm{Pb}$ and $\mathrm{p}-\mathrm{Pb}$ collisions at top SPS energy $\left(\sqrt{s}_{\mathrm{NN}}=17.2 \mathrm{GeV}\right)$, as observed by the WA97-NA57 collaboration [3]. Also, it seems that this ratio increases quickly in heavy ion collisions as a function of centre-of-mass energy going from 1 to a few GeVs and stays constant thereafter.

The big question is of the origin of this observed strangeness enhancement. Is the original prediction of generation in the plasma and subsequent coalescence still viable? Or, rather, are the excess strange quarks produced essentially at hadronization? Or, finally, is strangeness 


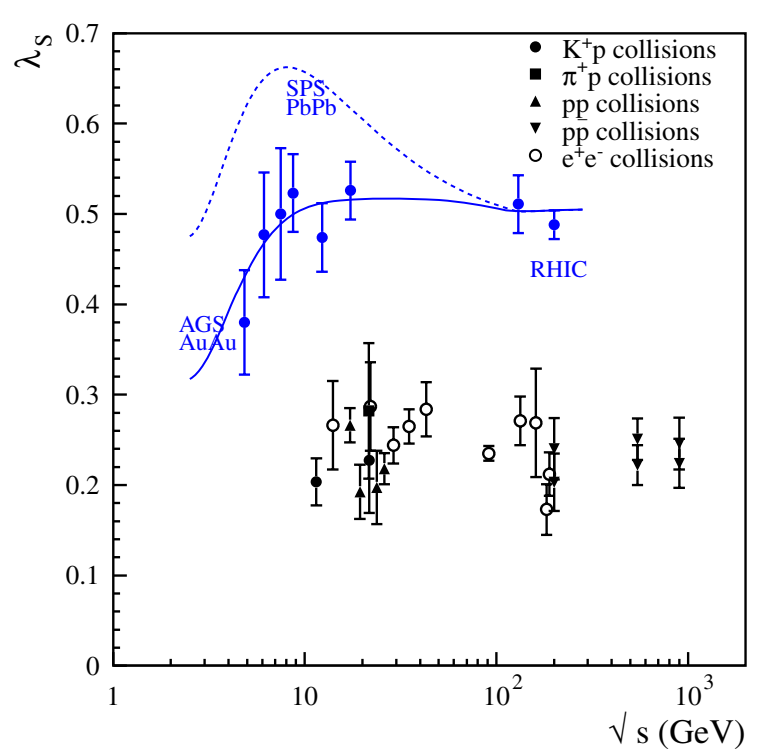

Figure 1. Wroblewski ratio in elementary and heavy ion collisions as estimated from statistical model fits. The superimposed dashed line is the predicted value from a fully equilibrated hadron gas, the solid line the interpolation from hadron gas with extra strangeness suppression $\gamma_{S}$.

produced during an intense hadronic re-scattering stage, according to transport models ansatz? Before trying to answer these questions, it is necessary to address a preliminary very important issue, i.e. whether we have produced a completely equilibrated hadron gas or not. If we have a completely equilibrated hadron gas, strangeness content is completely determined and gives information on freeze-out state, but it is not a probe of earlier stages of the process. Solving this problem may have a considerable impact on our understanding of strangeness production in relativistic heavy ion collisions.

\section{Statistical model and strangeness undersaturation}

The main tool to probe the formation of an equilibrated hadron gas is the fits of the measured particle multiplicities or ratios to the statistical model, that is the ideal hadron-resonance gas. Many authors have performed such analyses trying to pinpoint the thermodynamical parameters of the hadron emitting source at the chemical freeze-out and their conclusions are vastly different in this respect. Some [4] conclude that a completely equilibrated hadron gas has been produced throughout the examined centre-of-mass energy range (from low AGS to RHIC), others [5] that this never occurs. The reason for such a dramatic difference in physical conclusions resides on one hand on data selection and, on the other hand, on parameter choice in fitting procedure.

The conclusion that a completely equilibrated hadron gas is found relies on the use of midrapidity densities as experimental input compared with integrated yields of a single fireball at full hadrochemical equilibrium as a theoretical model. The underlying idea is that, being the QGP fireball expected at midrapidity, its properties can be probed by using midrapidity densities. However, such an approach requires the existence of a region around midrapidity (a plateau) where the thermodynamical parameters do not vary much. Conversely, if the 

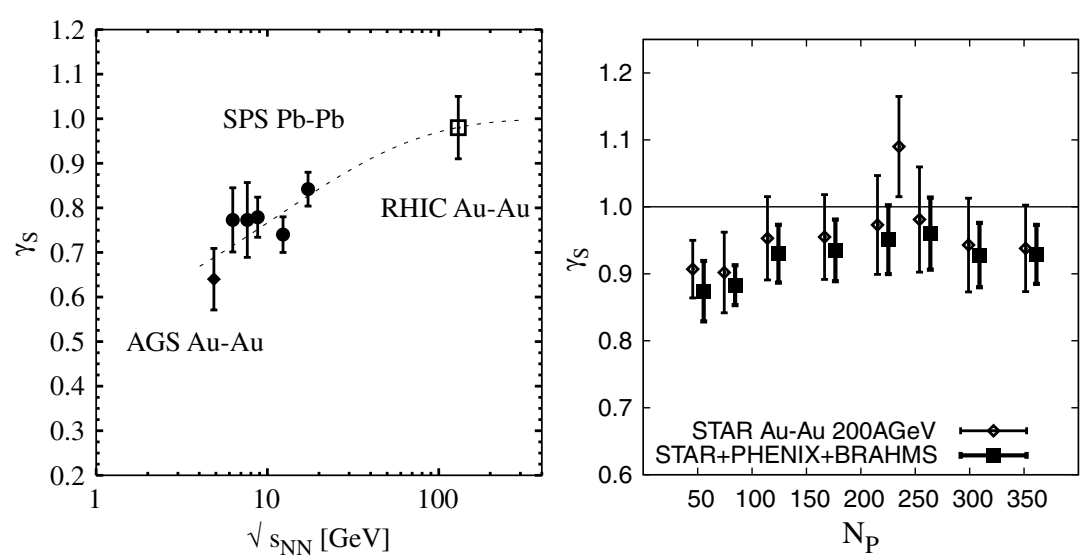

Figure 2. Left panel: $\gamma_{S}$ as a function of centre-of-mass energy in central heavy ion collisions (from [6]). Right panel: $\gamma_{S}$ as a function of centrality in Au-Au collisions at $\sqrt{s}_{\mathrm{NN}}=200 \mathrm{GeV}$ in central heavy ion collisions [7]

observed particle rapidity distributions are not sufficiently wider than that of a single fireball at the thermal freeze-out temperature, the use of midrapidity densities instead of integrated multiplicities artificially enhances heavier particles which have, in general, narrower rapidity width than lighter particles. This has two biasing effects: increasing the estimated temperature and enhancing the yield of particles carrying strange quarks which are generally heavier than the non-strange ones [8] so that the strangeness undersaturation parameter $\gamma_{S}$ turns out to be approximately 1 in these fits and essentially unnecessary.

In fact, up to SPS energies, the rapidity distributions are not wide enough to allow the use of midrapidity densities. For instance, the pion rapidity distribution at $\sqrt{s}_{\mathrm{NN}}=17.2 \mathrm{GeV}$ has a dispersion width of 1.3 , while that of a single fireball at rest with $T=125 \mathrm{MeV}$ (the thermal freeze-out temperature) is about 0.8 , hence not much smaller. Conversely, the measured width at RHIC at $\sqrt{s}_{\mathrm{NN}}=200 \mathrm{GeV}$ is 2.1 , which is reasonably larger than 0.8 . Thus, the use of midrapidity densities allows us to determine the thermodynamical parameters of the average fireball at midrapidity at RHIC energies onwards (roughly from $100 \mathrm{GeV}$ ), but not at SPS and lower energies. Therein, fits to full phase space yields provide a more appropriate, though amendable, estimate of the chemical freeze-out parameters. For recent studies including rapidity- dependent chemical potentials see $[9,10]$.

As a consequence, a strangeness undersaturation parameter $\gamma_{S}<1$ is needed to describe particle multiplicities in central heavy ion collisions. This parameter shows an increasing trend from AGS to RHIC, where it attains its maximal value 1 (see figure 2). Moreover, a $\gamma_{S}<1$ is also needed in peripheral collisions at RHIC for midrapidity densities, as shown in figure 2 .

\section{Canonical suppression}

It has been argued [11] that the observed strangeness undersaturation (i.e. $\gamma_{S}<1$ ) at energies lower than SPS is owing to the so-called canonical suppression effect. Namely, strange particles are further suppressed in pp collisions and peripheral relativistic heavy ion collisions with respect to their expected yield in a grand-canonical ensemble (or thermodynamic limit) 


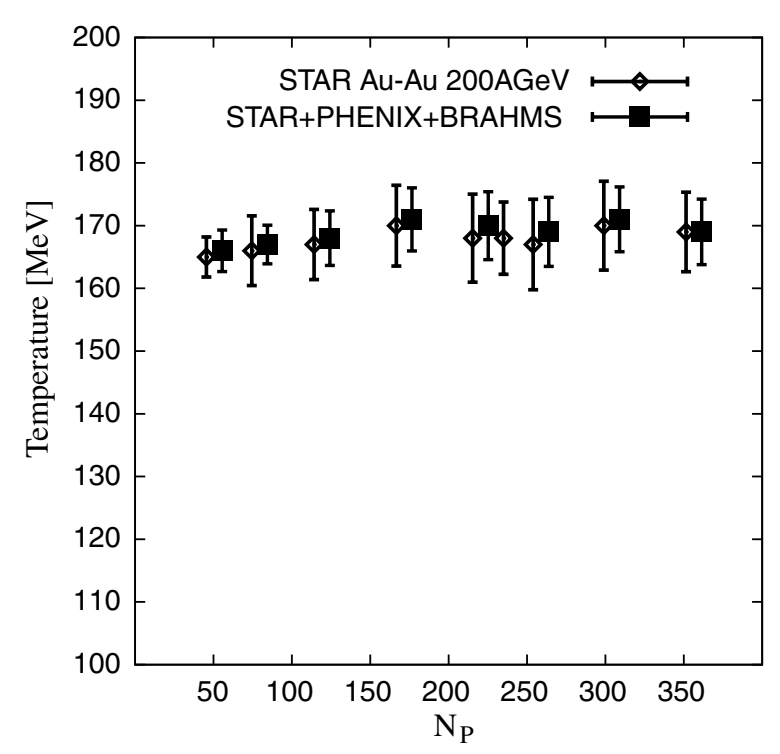

Figure 3. Chemical freeze-out as a function of centrality in $\mathrm{Au}-\mathrm{Au}$ collisions at $\sqrt{s}_{\mathrm{NN}}=$ $200 \mathrm{GeV}$ [7].

because strangeness is exactly vanishing within a small volume, called strangeness correlation volume (SCV), not necessarily coinciding with the global volume. Therefore, going from pp collisions to central heavy ion collisions through peripheral ones, one expects to observe a relative enhancement of strange particles due to approaching the thermodynamic limit, which is hierarchical: $\Omega$ yield increases faster than $\Xi$ which increases faster than $\Lambda$ or kaons. Yet, although this hierarchy of enhancements is observed (see figure 5), neither SPS nor RHIC have observed the saturation which should be there if the SCV attains a sufficiently large value. In fact, this means that the SCV only reaches its saturation value (the one sufficient for the system to be essentially grand-canonical) at RHIC precisely in central collisions, where $\gamma_{S} \simeq 1$. This would be quite a striking coincidence. Therefore, we think that canonical suppression is quite an unnatural explanation of the data, as already pointed out in [12].

The best probe to investigate the phenomenon of strangeness undersaturation is indeed the $\phi$ meson. This is not an open strange particle, thus it is not canonically suppressed, yet,

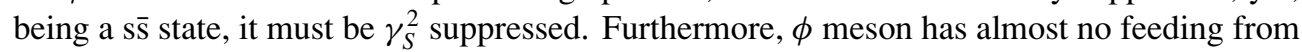
heavier light-flavoured species and its production is entirely direct.

It was pointed out quite early [13] that a statistical model with canonical suppression mechanism, i.e. with SCV as additional parameter, would have not been able to explain the deviation of the $\phi$ meson yield from its grand-canonical value and this has been demonstrated in fits to NA49 multiplicities [8]. Recently, the STAR collaboration has measured the midrapidity densities of $\phi$ meson very accurately and the observed pattern as a function of centrality clearly shows (see figure 5) that these do not scale linearly with the number of participants, rather the ratio to pp value increases rapidly at very peripheral collisions slowly saturating thereafter. This nonlinear increase cannot be attributed to a variation of the chemical freeze-out temperature because this is astonishingly constant as a function of $N_{p}$ as shown in figure 3 and proves that a genuine extra suppression related to the strange quark is needed, as also reflected in the $\gamma_{S}$ fitted value (see figure 2). 


\section{Core-corona superposition}

Can we explain $\gamma_{S}$ in relativistic heavy ion collisions in more fundamantal terms? A few years ago [8] Stock proposed that $\gamma_{S}<1$ in global fits could be the effect of superposing a completely equilibrated hadron gas $\left(\gamma_{S}=1\right)$ originated from the core of the nuclear collision (i.e. the hadronization of the plasma) to a corona of single NN collisions where the particle readily escapes the interaction region. Since strangeness is largely suppressed in NN collisions with respect to the grand-canonical value while the temperature is almost the same as we know from pp statistical model analysis [6, 14], if the number of such single NN collisions accounts for a significant fraction of total particle production, a global fit to one hadron-resonance gas would actually find $\gamma_{S}$ significantly less than 1 . Indeed, this idea proved to be able to satisfactorily reproduce particle multiplicities in central $\mathrm{C}-\mathrm{C}, \mathrm{Si}-\mathrm{Si}$ and $\mathrm{Pb}-\mathrm{Pb}$ collisions at top SPS energy.

This core-corona superposition mechanism has been invoked by Bozek a few years ago [15] to reproduce the $K / \pi$ ratio as a function of centrality in Au-Au collisions and has recently been advocated in a paper by Werner [16] to be capable of explaining some more otherwise 'mysterious' effects. A sharp superposition of a completely equilibrated hadron gas with $\mathrm{NN}$ collisions is indeed a zero-order approximation as the actual process is certainly more complex with those two extremes continuously linked through intermediate steps and indeed in [16] a more general concept of corona has been used, defined as a 'dilute' peripheral region distinguished from the 'dense' region in the core. Yet, this simple superposition scheme can be a very useful one to understand the physics of particle production. Accordingly, the rapidity density at midrapidity of any particle species is given by:

$$
\left\langle\frac{\mathrm{d} n}{\mathrm{~d} y}\right\rangle=N_{s}\left\langle\frac{\mathrm{d} n}{\mathrm{~d} y}\right\rangle_{p p}+f\left(V_{0}-\delta V_{0}\right)\left\langle\frac{\mathrm{d} \rho}{\mathrm{d} y}\right\rangle_{\text {core }}
$$

where $N_{s}$ is the mean number of single NN collisions, $V_{0}$ is the initial volume of the initial nuclear overlapping region, $\delta V_{0}$ is its thin outer shell where these single $\mathrm{NN}$ collisions occur, $f$ is the growth factor (i.e. how much this volume expands up to chemical freeze-out) and $\mathrm{d} \rho / \mathrm{d} y$ is the particle density per unit rapidity in the core relevant to a completely equilibrated hadron gas, i.e. with $\gamma_{S}=1$. Dividing by the number of wounded nucleons $N_{W}{ }^{3}$ and the rapidity density in pp, we obtain a simple expression from (1):

$$
\frac{\left\langle\frac{\mathrm{d} n}{\mathrm{~d} y}\right\rangle}{N_{W}\left\langle\frac{\mathrm{d} n}{\mathrm{~d} y}\right\rangle_{p p}} \simeq A+\frac{N_{s}}{N_{W}}(1-2 A)
$$

where $A$ is an unknown constant. This expression fulfils the constraint that both the left-and right-hand side ought to be $1 / 2$ when $N_{W}=2$ and $N_{s}=1$. Remarkably, for the $\phi$ meson the constant $A$ is independent of $N_{W}$ because $T$ is in fact independent of centrality (see figure 3 ) and $\phi$ does not suffer possible canonical suppression. Indeed, $A$ is the asymptotic value of the normalized yield, when the number of participants becomes very large; hence, it is normally larger than $1 / 2$ and the second term of the right-hand side of equation (2) is negative.

The problem now is how to define and estimate the number $N_{s}$ of single NN collisions. Ideally, we would like them to be those independent collisions where produced particles do not reinteract at all with the surrounding environment. In perfectly central collisions, they supposedly are single NN collisions where both nucleons undergo exactly one collision occurring at the edge of the overlap region. On the other hand, in extreme peripheral nuclear collisions, they should reduce to one NN collision. In all other cases, they are tightly related

3 In this work wounded and participant nucleons are synonymous. 


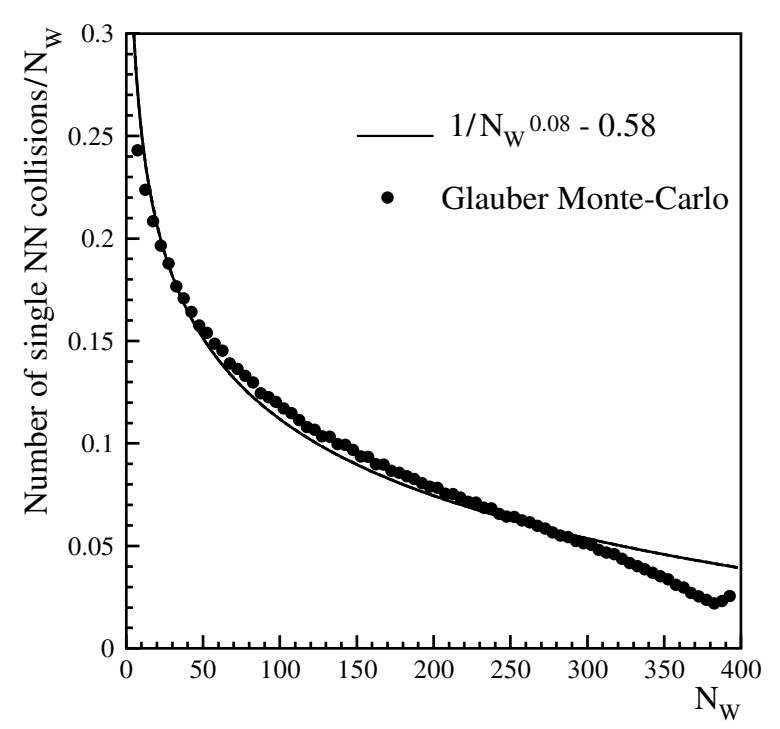

Figure 4. Number of single $\mathrm{NN}$ collisions divided by the number of wounded nucleons as a function of number of wounded nucleons according to Glauber Monte Carlo. The solid line is the interpolation (4).

to the NN collisions occurring at the edge of the overlap region where only one nucleon from either nucleus is involved, but their number cannot be defined in a clearcut way without a full dynamical model of the collision. However, we can resort to a definition interpolating the perfectly central and the extreme peripheral case and relying on the Glauber model. Such a definition might be:

$$
N_{s} \equiv \min \left[N_{1(a)}, N_{1(b)}\right],
$$

where $N_{1(a)}\left(N_{1(b)}\right)$ is the number of nucleons colliding once according to the Glauber model. We estimated the thus-defined $N_{s}$ as a function of centrality by means of a Glauber Monte Carlo calculation. The resulting $N_{s} / N_{W}$ ratio can reasonably be fitted for $N_{W}>10$ by (see figure 4):

$$
\frac{N_{s}}{N_{W}}=\frac{1}{N_{W}^{0.08}}-0.58
$$

Plugging (4) into (2) we have an expression of the normalized yield as a function of the number of wounded nucleons, that is centrality, depending on one unknown parameter $A$. This can be determined by matching the model with the measured value in the most central bin and then the centrality evolution is completely determined. The obtained curve is in impressive agreement with the data, as shown in figure 5; the formula matches the experimental points to a high degree of accuracy. This is a clear evidence that the envisaged core-corona superposition is able to account for the strangeness undersaturation phenomenon.

The same exercise can be repeated for open strange particles, the result being shown in figure 5. It can be seen that the curves match the data in the most central bins, while they overestimate the measured points in most peripheral bins: this is likely due to the canonical suppression effect in the core which is not taken into account in the formula (2) but should indeed show up for peripheral collisions. Finally, we observe that other definitions of $N_{s}$ are possible (e.g. $\left.\left(N_{1(a)}+N_{1(b)}\right) / 2\right)$ but they lead to similar results. 

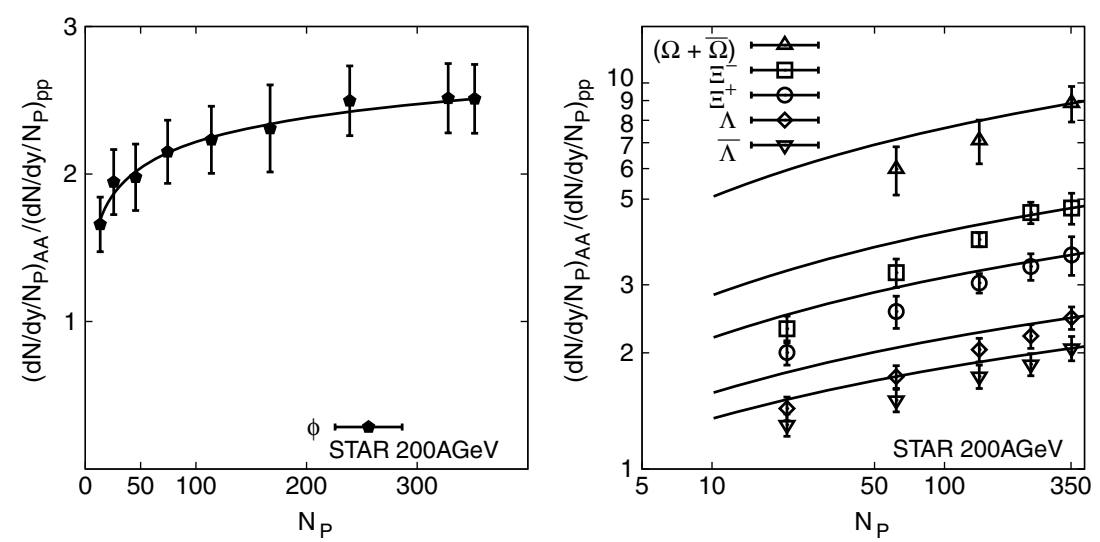

Figure 5. $\phi$ (left panel) and hyperons (right panel) rapidity density per wounded nucleon as a function of participants normalized to pp collisions. Data points from STAR [17]; solid lines are the predictions from core-corona superposition (see the text).

\section{Discussion and conclusions}

If our interpretation of $\phi$ production as a function of centrality is correct, several remarkable consequences are implied. Firstly, the enhancement of relative strange particle production going from peripheral to central collisions is mainly due to a geometrical effect of core-corona superposition. Canonical suppression plays a role only in the most peripheral collisions and it is possible that the SCV simply coincides with the core volume. Secondly, the $\phi$ data support evidence for a completely equilibrated hadron gas in the core throughout all centralities at RHIC, whose temperature is constant and equal to $165 \mathrm{MeV}$.

The same conclusion is also likely to apply to SPS. The fact that there $\gamma_{S} \simeq 0.85$ in central collisions [6], significantly lower than at RHIC, is related to the lower weight of the core compared to the corona. Indeed, as energy decreases, so does the freeze-out volume of the core and the multiplicity of particles stemming from it, while the number of single NN collisions decreases only slightly, the NN cross section being slowly varying. This would nicely explain the mild increase of $\gamma_{S}$ as a function of centre-of- mass energy (see figure 2); nevertheless, a complete reanalysis of the data is compelling. As has been mentioned, early analyses of central collisions at top SPS energy based on this picture were fairly succesful [8], but peripheral collisions are indespensable to confirm this idea. In this respect, NA49 is going to update preliminary measurements [18] which were used to determine $\gamma_{S}$ in peripheral $\mathrm{Pb}-\mathrm{Pb}$ collisions [19].

The constancy of $T$ as a function of centrality (see figure 3) which was first observed by the STAR collaboration [20] is confirmed in our analysis of RHIC data [7] to a high degree of accuracy. This stunning independence of centrality is hard to reconcile with collisional thermalization, as pointed out in [21] as it would require a dramatic dependence of hadronic reaction rates on temperature. Also, the $\Omega$ yield is very difficult to reproduce in such an approach [22] unless invoking the existing massive resonant degrees of freedom [23].

\section{Acknowledgments}

We are greatly indebted with $\mathrm{H}$ Caines and J H Chen for providing us with preliminary STAR measurements of particle production in peripheral $\mathrm{Au}-\mathrm{Au}$ collisions. We warmly thank STAR 
Collaboration for allowing us to use these data. We are grateful to P Bozek, H Caines and K Werner for useful comments. F Becattini would like to express his gratitude to the organizers of the quark matter conference.

\section{References}

[1] Rafelski J and Muller B 1982 Phys. Rev. Lett. 481066

[2] Becattini F, Gazdzicki M, Keranen A, Manninen J and Stock R 2004 Phys. Rev. C 69024905

[3] Antinori F (WA97 Collaboration) et al 1998 Phys. Lett. B 433209

[4] Andronic A, Braun-Munzinger P and Stachel J 2006 Nucl. Phys. A 772167

[5] Rafelski J, Letessier J and Torrieri G 2001 Phys. Rev. C 64054907

[6] Becattini F, Manninen J and Gazdzicki M 2006 Phys. Rev. C 73044905

[7] Becattini F and Manninen J (in preparation)

[8] Becattini F, Gazdzicki M, Keranen A, Manninen J and Stock R 2004 Phys. Rev. C 69024905

[9] Biedron B and Broniowski W 2007 Phys. Rev. C 75054905

[10] Becattini F and Cleymans J 2007 J. Phys. G: Nucl. Part. Phys. 34 S959

[11] Hamieh S, Redlich K and Tounsi A 2000 Phys. Lett. B 48661

[12] Xu N 2007 Talk Given at Critical Point and Onset of Deconfinement (GSI, Darmstadt, 9-13 July)

[13] Sollfrank J, Becattini F, Redlich K and Satz H 1998 Nucl. Phys. A 638 399C

[14] Becattini F and Passaleva G 2002 Eur. Phys. J. C 23551

[15] Bozek P 2005 Acta Phys. Polon. B 363071

[16] Werner K 2007 Phys. Rev. Lett. 98152301

[17] Chen J H (for the STAR Collaboration) 2008 J. Phys. G: Nucl. Part. Phys. 35104053

[18] Gazdzicki M 2008 private communication

[19] Becattini F, Maiani L, Piccinini F, Polosa A D and Riquer V 2006 Phys. Lett. B 632233

[20] Adams J (STAR Collaboration) et al 2004 Phys. Rev. Lett. 92112301

[21] Heinz U and Kestin G 2006 Universal chemical freeze-out as a phase transition signature PoS C POD2006 038 (Preprint nucl-th/0612105)

[22] Kapusta J I 2004 J. Phys. G: Nucl. Part. Phys. 30 S351

[23] Noronha-Hostler J, Greiner C and Shovkovy I A 2007 (Preprint arXiv:0711.0930) 